\title{
Endoscopic full-thickness resection in the colorectum with a novel over-the-scope device: first experience
}

Authors

Institutions
Arthur Schmidt ${ }^{1,}$ *, Peter Bauerfeind ${ }^{2}$,, Christoph Gubler ${ }^{2}$, Michael Damm¹, Markus Bauder ${ }^{1}$, Karel Caca $^{1}$

Department of Gastroenterology and Oncology, Klinikum Ludwigsburg, Ludwigsburg, Germany

2 Department of Gastroenterology and Hepatology, Zürich, Switzerland
Bibliography

Dol http://dx.doi.org/

10.1055/s-0034-1391781

Published online: 12.3.2015

Endoscopy 2015; 47: 719-725

(c) Georg Thieme Verlag KG

Stuttgart · New York

ISSN 0013-726X

\section{Corresponding author}

Karel Caca, MD, PhD

Klinikum Ludwigsburg

Medizinische Klinik I

Posilipo-Strasse 1-4

71640 Ludwigsburg

Germany

Fax: +49-7141-9964219

karel.caca@kliniken-lb.de

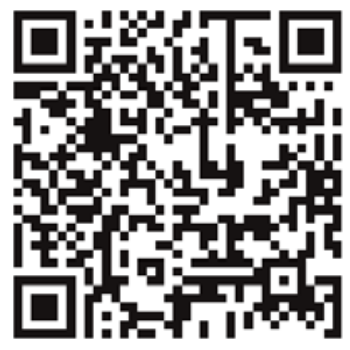

Scan this QR-Code to watch the video comment.
Background and study aims: Endoscopic fullthickness resection (EFTR) in the lower gastrointestinal tract may be a valuable therapeutic and diagnostic approach for a variety of indications. Although feasibility of EFTR has been demonstrated, there is a lack of safe and effective endoscopic devices for routine use. The aim of this study was to investigate the efficacy and safety of a novel over-the-scope device for colorectal EFTR. Patients and methods: Between July 2012 and July 2014, 25 patients underwent EFTR at two tertiary referral centers. All resections were performed using the full-thickness resection device (FTRD; Ovesco Endoscopy, Tübingen, Germany). Data were collected retrospectively.

Results: Indications for EFTR were: recurrent or incompletely resected adenoma with nonlifting sign $(n=11)$, untreated adenoma and nonlifting $\operatorname{sign}(\mathrm{n}=2)$, adenoma involving the appendix $(\mathrm{n}=$ 5), flat adenoma in a patient with coagulopathy $(n=1)$, diagnostic re-resection after incomplete

\section{Introduction}

$\nabla$

Endoscopic mucosal resection (EMR) and endoscopic submucosal dissection (ESD) are well-investigated, minimally invasive therapies for colorectal neoplasms [1-4]. The rate of local recurrence after EMR of advanced nonpedunculated adenomas has been reported to be $16 \%$ in a recent prospective, multicenter study [4]. Endoscopic retreatment of these lesions can be challenging because of scaring and fibrosis $[2,3]$. Hence, endoscopic full-thickness resection (EFTR) may be an adequate approach for diagnostic or therapeutic tissue removal in these cases. In addition, the technique may be indicated for previously untreated nonlifting lesions or colorectal neoplasms at difficult anatomic locations, rendering surgery

\footnotetext{
* These authors contributed equally to this work.
}

resection of a T1 carcinoma $(\mathrm{n}=2)$, adenoma involving a diverticulum $(n=1)$, submucosal tumor $(\mathrm{n}=2)$, and diagnostic resection in a patient with suspected Hirschsprung's disease $(n=1)$. In one patient, the lesion could not be reached because of a sigmoid stenosis. In the other patients, resection of the lesion was macroscopically complete and en bloc in 20/24 patients (83.3\%). The mean diameter of the resection specimen was $24 \mathrm{~mm}$ (range $12-40 \mathrm{~mm}$ ). The $\mathrm{R} 0$ resection rate was $75.0 \%(18 / 24)$, and full-thickness resection was histologically confirmed in $87.5 \%$. No perforations or major bleeding were observed during or after resection. Two patients developed postpolypectomy syndrome, which was managed with antibiotic therapy.

Conclusions: Full-thickness resection in the lower gastrointestinal tract with the novel FTRD was feasible and effective. Prospective studies are needed to further evaluate the device and technique.

unnecessary. In cases of invasive early carcinomas, EFTR may increase the diagnostic yield and help to determine the most appropriate therapy for the patient. EFTR may also be indicated for diagnostic purposes in patients with neurogenic, colonic, motility disorders, such as Hirschsprung's disease. However, to date, colonic EFTR is not an established technique in clinical practice because there is a lack of safe and effective resection devices and techniques.

Endoscopic resection of lesions by application of an over-the-scope clip (OTSC) followed by snare resection has been described to be feasible [57]. The full-thickness resection device (FTRD; Ovesco Endoscopy, Tübingen, Germany) is a novel, over-the-scope device, which consists of a modified OTSC mounted on a long cap with a preloaded snare. It is an instrument for flexible EFTR and diagnostic tissue acquisition of suitable lesions in the colon and rectum. The device has 


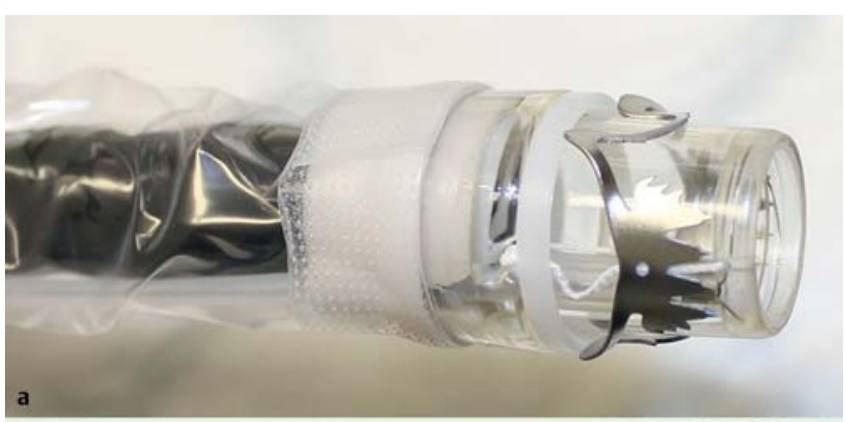

\section{Description of device}

The FTRD is a pre-assembled over-the-scope device ( $\bullet$ Fig. 1). A transparent cap with a modified 14-mm OTSC is mounted over a standard colonoscope. The cap has an inner diameter of $13 \mathrm{~mm}$ and a length of $23 \mathrm{~mm}$ (measured from the tip of the endoscope). A monofilament 14 -mm polypectomy snare is preloaded onto the tip of the cap. The snare is not advanced through the working channel but rather runs along the outer surface of the endoscope under a plastic sheath. This sheath is fixed onto the cap and pulled over the scope shaft after mounting the cap.

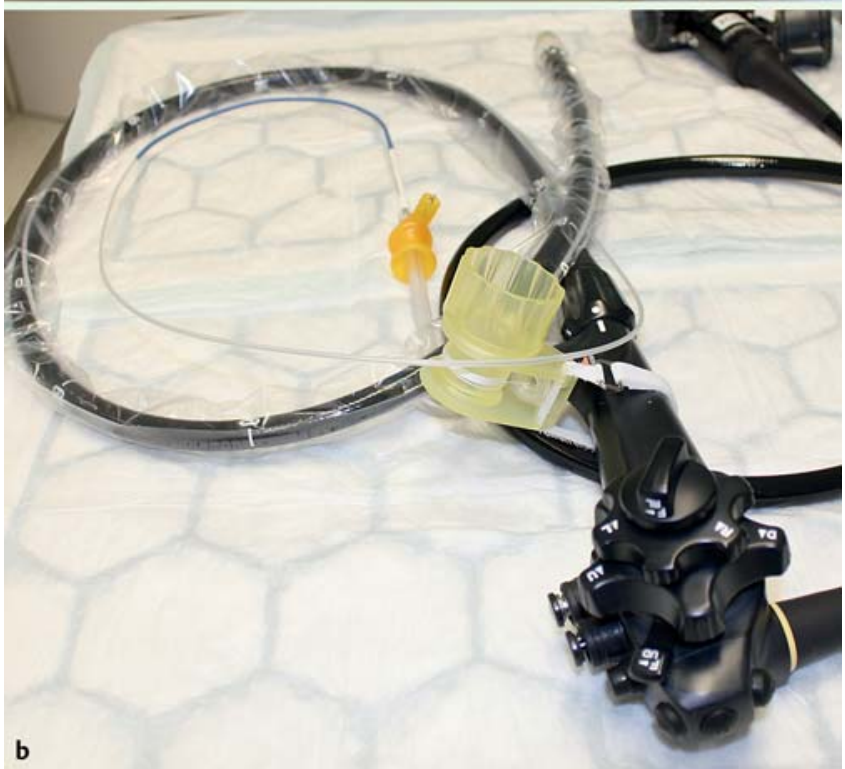

Fig. 1 The full-thickness resection device (FTRD; Ovesco Endoscopy, Tübingen, Germany). a A modified 14-mm over-the-scope clip is mounted onto a long transparent cap. b The FTRD assembled on a standard colonoscope.

been investigated in preclinical trials. Moreover, a small recently published series demonstrated successful resection of recurrent adenomas in three patients, and a case report described resection of an adenoma arising from a diverticulum [8-13]. The current report describes the clinical experience from two centers using this device for EFTR in the lower gastrointestinal (GI) tract.

\section{Patients and methods \\ $\nabla$}

\section{Study design and patients}

This study was a retrospective study conducted at two European tertiary referral centers. Between July 2012 and July 2014, 25 patients underwent EFTR using the FTRD. Outcome measures were: 1) technical success (target lesion resected en bloc and macroscopically complete); 2) R0 resection rate (proportion of patients with negative lateral and deep margins on histological examination); 3) full-thickness resection rate (proportion of patients with histologically confirmed full-thickness resection); 4) adverse event rates.

Written informed consent was obtained from all patients. The study was performed in accordance with the declaration of Helsinki. The FTRD was provided by Ovesco Endoscopy, Tübingen, Germany. The study was not funded.

The device has a Conformité Européenne mark and is commercially available in Europe. It has not yet been approved by the Food and Drug Administration.

\section{Resection technique}

The endoscope with the mounted FTRD is introduced into the colon and the target lesion is identified. A grasping forceps or a tissue anchor (Ovesco Endoscopy) is advanced through the working channel of the endoscope to grasp the lesion. The lesion is slowly pulled into the cap in order to capture a double full-thickness layer of the colonic wall. With the lateral margins of the lesion pulled into the cap, the OTSC is deployed. The pseudopolyp created by the OTSC is then resected using the pre-loaded snare while the OTSC secures integrity of the colonic wall (for schematic illustration of the technique, see Fig. 2).

\section{Procedures and periprocedural management}

Before resection, all patients underwent physical examination, and blood tests were obtained to check for coagulopathy. Preoperative endoscopic ultrasound and/or computed tomography (CT) were only performed in patients with malignant (T1) lesions to exclude deep wall infiltration and metastatic disease.

All procedures were performed under deep sedation (midazolam bolus and continuous propofol infusion) without endotracheal intubation. Blood pressure, electrocardiogram, and oxygen saturation were constantly monitored during the procedure. All patients received prophylactic antibiotic therapy (intravenous ciprofloxacin and metronidazole twice a day for 3 days starting immediately before the procedure). All procedures were performed by experienced endoscopists.

Prior to resection, the target lesion was identified with a standard colonoscope. In the majority of patients, the lateral margins were marked using argon plasma coagulation; in case of flat adenomas, staining with tolouidine blue was performed. The endoscope was extracted from the patient and the FTRD was mounted onto the scope. The endoscope with the mounted FTRD was reintroduced into the patient and advanced to the target lesion. The lesion was then resected as described above and the endoscope was extracted with the resection specimen in the cap. The FTRD was removed from the scope, and after re-inserting the endoscope, the resection site was inspected ( $\bullet$ Fig. 3, Video 1 ).

After the procedure, patients were hospitalized for at least two nights and were closely monitored for clinical signs of bleeding (hematochezia) or perforation (daily abdominal examination). Abdominal radiography was performed in patients with postprocedural clinical signs of perforation or peritonitis only. Patients were started on clear liquids 3-6 hours after the procedure if there were no signs of peritonitis. A normal diet was usually started the following day (postoperative Day 1). 


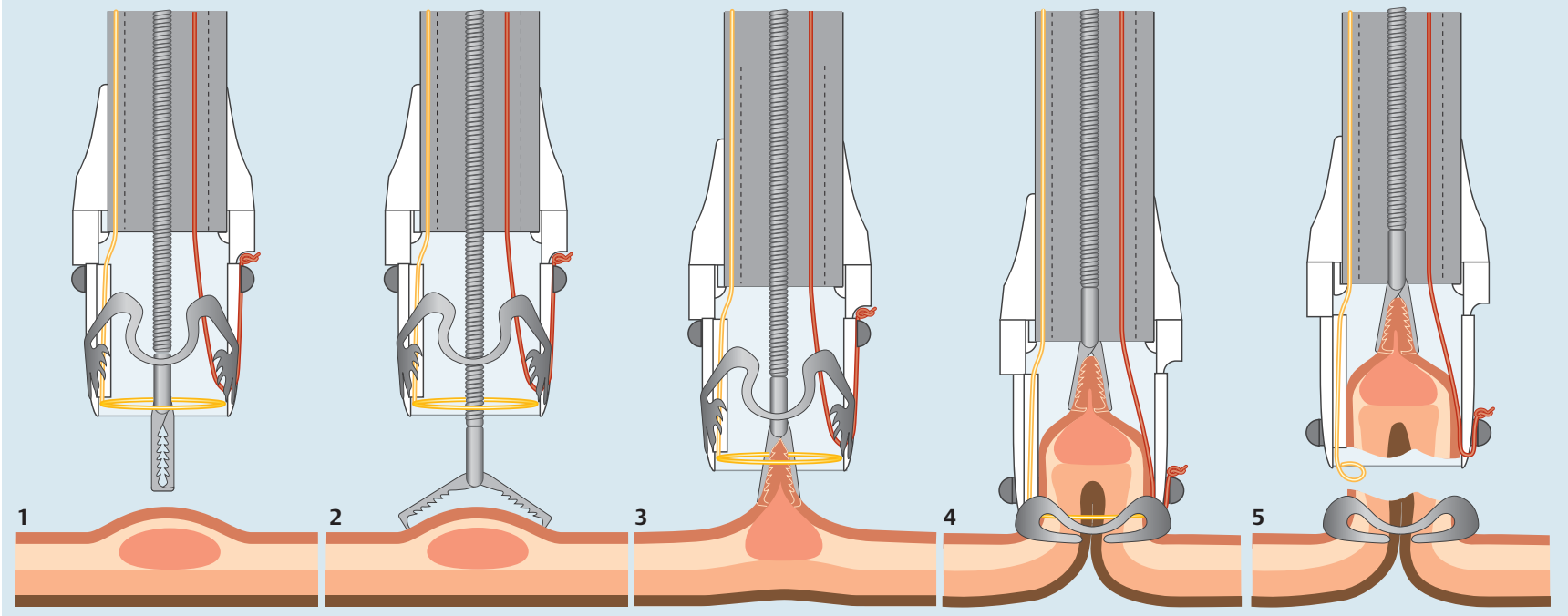

Fig. 2 Schematic illustration of the resection procedure. 1 and 2: A grasping forceps is advanced through the working channel of the endoscope. 3: The target lesion is grasped and pulled into the cap. 4: The over-the-scope clip is deployed and creates a full-thickness duplication of the colonic wall. 5: The pseudopolyp above the clip is resected using the preloaded snare.

\section{Follow-up}

Patients were scheduled for the first endoscopic follow-up within 3-6 months after resection. Further follow-ups were scheduled individually depending on histology and endoscopic findings.

\section{Results}

\section{$\nabla$}

\section{Patient characteristics and indications for EFTR}

Between July 2012 and July 2014, 25 Patients underwent EFTR using the FTRD at two academic tertiary referral centers. The mean age was 70 years (range $43-84$ years). In the majority of patients, the indication for EFTR was a nonlifting adenoma (13/25), which included nine patients with recurrent adenomas, two patients with incompletely resected adenomas, and two patients with previously untreated adenomas. One additional patient had a flat (lifting) adenoma but increased risk of bleeding because of coagulopathy. Six patients had adenomas at difficult anatomic locations, including five patients with lesions involving the appendix and one patient with an adenoma involving a diverticulum. Diagnostic EFTR was performed in two patients with incompletely resected $\mathrm{T} 1$ carcinoma and in one patient with suspected Hirschsprung's disease. Two patients had colonic submucosal tumors, which were located in the ascending and in the descending colon. For detailed patient characteristics, location and size of the target lesions, and pre-procedural histology see $\bullet$ Table 1.

\section{Procedural data, technical success, and histopathology} The median procedure time was 50 minutes (range 10-177 minutes). This included advancing the scope to the lesion, marking the lesion, advancing the endoscope with the mounted FTRD, resection, reintroducing the scope without the FTRD, and inspection of the resection site.

In a patient with history of diverticulitis, the target lesion could not be reached because the FTRD could not be advanced beyond a sigmoid stenosis. Lesions in the remaining 24 patients were reached using the FTRD, and in 20 of the lesions resection was macroscopically complete and en bloc. Hence, the technical suc- cess rate was $83.3 \%$. The reasons for technically unsuccessful resection were an adenoma at the appendix $(n=2)$, which prevented complete en bloc resection with the FTRD (the preloaded snare did not close properly after deployment of the OTSC $[n=1]$; the lesion could not be pulled completely into the cap [n=1]). In these two cases, the FTRD was retrieved and the residual adenoma above the OTSC was completely resected using a standard monofilament snare after reintroducing the endoscope. Histology was classified as "Rx" (indeterminate) for piecemeal resection. In a patient with a recurrent rectal adenoma, the rectal wall could not be pulled completely into the cap, which resulted in residual adenoma next to the OTSC. The tissue next to the OTSC was resected using a snare. In the fourth patient, who had recurrent adenoma in the ascending colon, the lesion could not be completely pulled into the cap because of fibrosis, resulting in residual adenoma above the OTSC; this was also resected using a snare.

The mean size of the resection specimen (maximum diameter, determined by the pathologist) was $24 \mathrm{~mm}$ (range $12-40 \mathrm{~mm}$ ) (- Table 2). In the 24 specimens, histologically complete resection (defined as lateral and deep margins free of adenoma/tumor cells) was confirmed in 18 cases (75.0\%). One patient with a lesion at the appendix had adenoma cells at the lateral resection margins. In another patient with a nonlifting lesion, histology after diagnostic resection revealed adenocarcinoma at the lateral resection margins.

Histology of the two patients with submucosal tumors revealed leiomyoma in one case and hamartoma in the other case. In the two patients with T1 carcinomas who underwent re-resection using the FTRD, histology did not show any remnant carcinoma or adenoma, and no further therapy was scheduled. In the patient with suspected Hirschsprung's disease, aganglionosis was not evident in the resection specimen.

\section{Adverse events}

Minor bleeding at the resection site was observed in one patient ( 0 Table 2). Hemostasis was achieved by endoscopic injection of epinephrine solution. No major immediate or delayed bleeding was observed, and no patient required blood transfusion. There was no perforation or need for emergency surgery. 


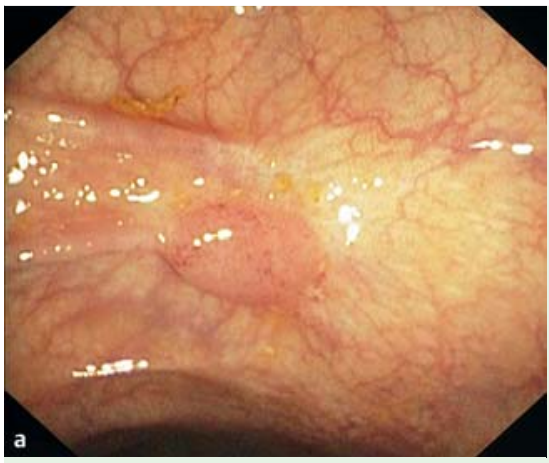

Fig.3 Full-thickness resection in a patient with a nonlifting, recurrent adenoma in the ascending colon (see also Video 1). a Endoscopic image of the nonlifting, flat, polypoid lesion. Note the scarring around the adenoma. b Endoscopic view with the mounted full-thickness resection device.

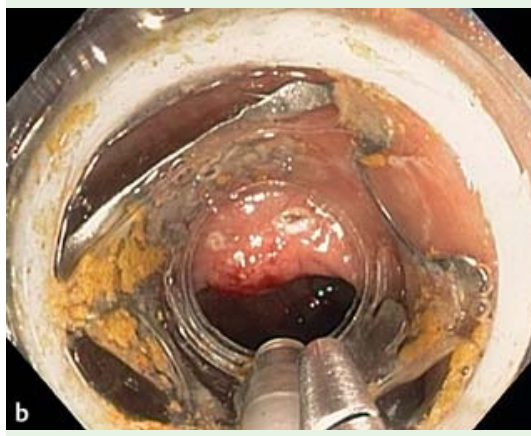
c Resection site showing the over-the-scope clip, which secures colonic wall patency. The resection was macroscopically complete.

d Resection specimen. e, f Histology showing the lateral margins of the adenoma ( $R 0$ resection). Both images illustrate full-thickness resection (whole muscu-

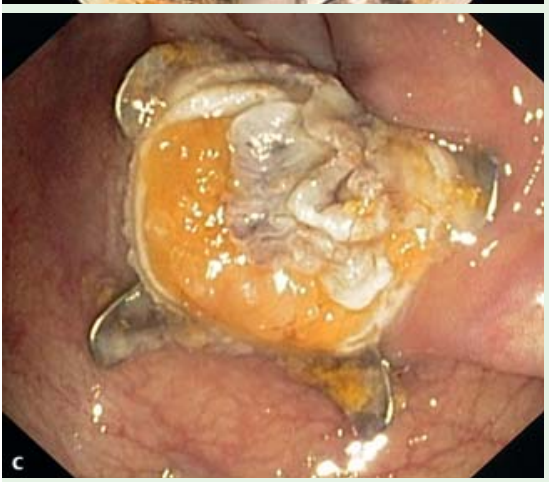
laris propria and serosa are visible).

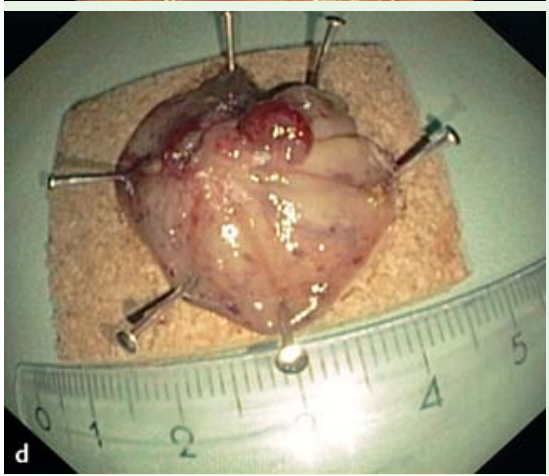

Two patients with adenomas in the cecum developed abdominal pain, fever, and elevation of C-reactive protein after the intervention. In both patients, colonic perforation was excluded by radiography. One of these patients also underwent a CT scan, which showed thickening of the cecal wall with no evidence of perforation, abscess, or appendicitis. Both patients were treated with intravenous antibiotics for 1 week and recovered completely after 3-4 days. No other adverse events were observed.

\section{Clinical outcome and follow-up:}

Endoscopic follow-up was available for 19 patients. In six patients, follow-up could not be obtained for the following reasons: one patient with known liver cirrhosis died 2 months after resection (liver failure); two patients underwent hemicolectomy for

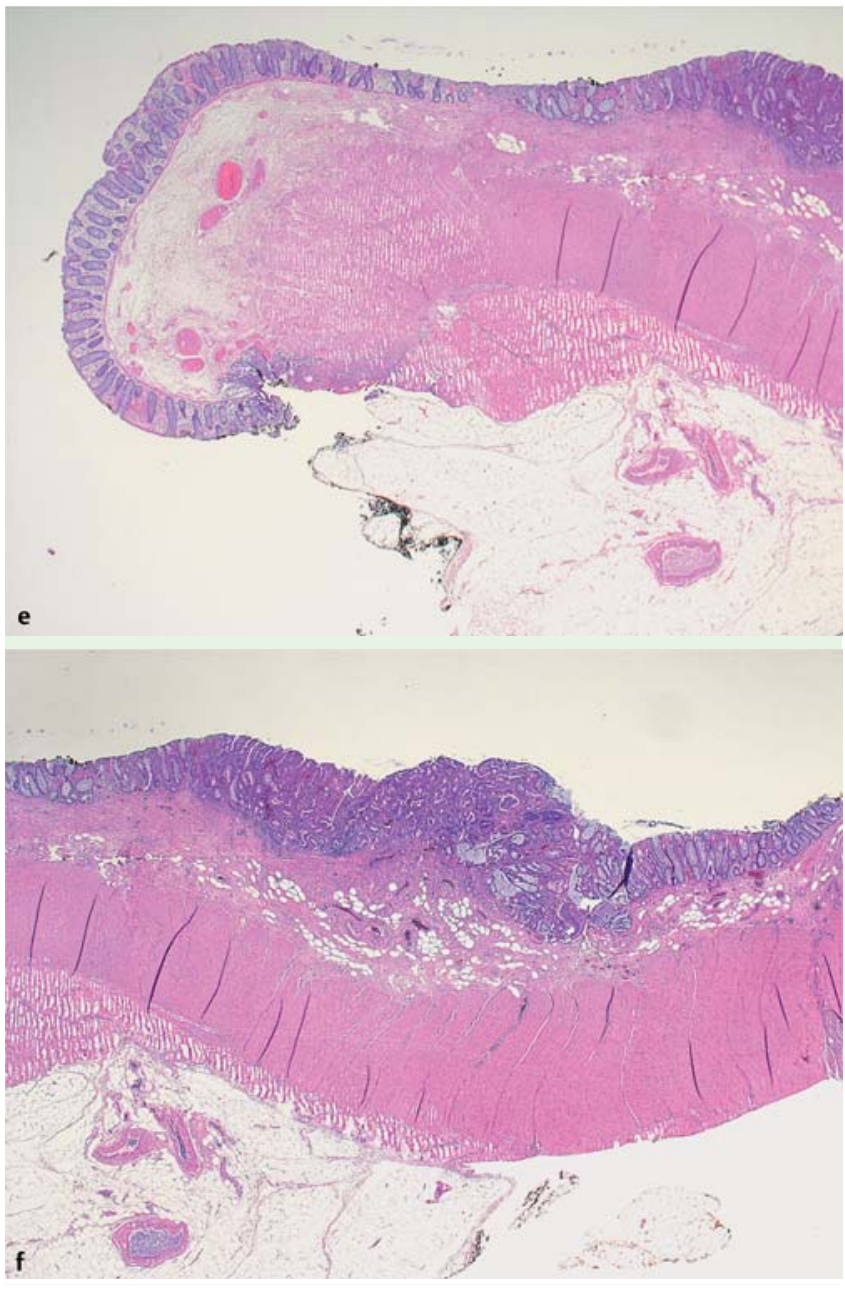

Video 1

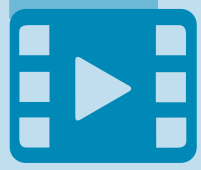

A 77-year-old woman was referred for resection of a recurrent adenom with high grade dysplasia in the transverse colon. Endoscopy showed a flat, polypoid, nonlifting lesion with an estimated diameter of $15 \mathrm{~mm}$. The lesion was marked circumferentially using coagulation. The endoscope with the mounted full-thickness resection device was then advanced to the transverse colon and the lesion was identified. A grasping forceps was used to gently pull the lesion into the cap. The over-thescope clip was deployed and the lesion was resected using the preloaded snare. Resection was macroscopically complete with no signs of bleeding or perforation. Histology of the full-thickness resection specimen confirmed complete lateral and deep resection (R0). 
Table 1 Patient characteristics $(n=25)$.

\begin{tabular}{|l|l|}
\hline Total no. of patients, $\mathrm{n}$ & 25 \\
\hline Age, mean (range), years & $70(43-84)$ \\
\hline Indication for EFTR, $\mathrm{n}(\%)$ & \\
\hline Recurrent adenoma with nonlifting sign & $9(36)$ \\
\hline Untreated adenoma with nonlifting sign & $2(8)$ \\
\hline Incompletely resected adenoma with nonlifting sign & $2(8)$ \\
\hline Flat adenoma in a patient with severe coagulopathy & $1(4)$ \\
\hline Diagnostic re-resection after incomplete resection of & \\
\hline T1 carcinoma & $2(8)$ \\
\hline Adenoma involving appendix & $5(20)$ \\
\hline Adenoma involving diverticulum & $1(4)$ \\
\hline Submucosal tumor & $2(8)$ \\
\hline Diagnostic EFTR for suspected Hirschsprung's disease & $1(4)$ \\
\hline Estimated diameter of lesions, mean (range), mm & $15.6(8-30)$ \\
\hline Histology prior to EFTR, $\mathrm{n}$ & \\
\hline Adenoma with low grade dysplasia & $10(40)$ \\
\hline Adenoma with high grade dysplasia & $10(40)$ \\
\hline Adenocarcinoma (T1) & $2(8)$ \\
\hline Histology not obtained & \\
\hline Resection site location, $\mathrm{n}(\%)$ & $3(12)$ \\
\hline Cecum & \\
\hline Ascending colon & $5(20)$ \\
\hline Transverse colon & $5(20)$ \\
\hline Descending colon & $3(12)$ \\
\hline Sigmoid & $4(16)$ \\
\hline Rectosigmoid transition & $2(8)$ \\
\hline Rectum & $3(12)$ \\
\hline end & $2(8)$ \\
\hline
\end{tabular}

EFTR, endoscopic full-thickness resection.

${ }^{1}$ Two patients with submucosal tumors, one with suspected Hirschsprung's disease.

adenocarcinoma with high risk features; one patient refused further follow-up; and two patients missed follow-up appointments and had to be re-scheduled. In 7 of 19 patients (36.8\%), the OTSC was in place at the first follow-up, but in 12 patients (63.2\%) it had dislodged spontaneously. The mean follow-up period was 127 days (range $43-372$ days).

In the patients with initial successful R0 resection, one recurrent adenoma was observed at the appendiceal orifice at 3-months' follow-up. This recurrence was not visible macroscopically but was diagnosed histologically in the biopsies taken from the resection site. This patient was scheduled for another EFTR (procedure not yet done at time of manuscript preparation). In one patient with a previously untreated nonlifting lesion, histology after EFTR showed a completely resected adenocarcinoma with invasion of lymphatic vessels (L1). This patient was referred to surgery for resection. The surgical resection specimen did not show any residual tumor.

The clinical courses of patients with macroscopically or histologically incomplete resection were as follows. Histology of the patient mentioned above with a nonlifting, previously untreated lesion in the descending colon showed adenocarcinoma. As one lateral margin of the resection specimen was not free of tumor cells (Rx) and the carcinoma showed deep (sm3) submucosal invasion, the patient was referred for hemicolectomy; histology of the resected colon, however, did not show any residual tumor or lymph node metastasis. The two patients with incomplete resection of adenomas at the appendix underwent endoscopic followup at 3 months. In both patients, the OTSC had fallen off; residual adenoma was successfully resected using forceps in one case and the FTRD in the other case. Further follow-up is still pending. Another patient with microscopically Rx resection (lateral margins) of an adenoma with low grade dysplasia at the appendix did not
Table 2 Outcome and adverse events $(n=25)$.

\begin{tabular}{|l|l|}
\hline Target lesion reached with FTRD, $\mathrm{n}(\%)$ & $24 / 25(96.0)$ \\
\hline Technical success, $\mathrm{n}(\%)$ & $20 / 24(83.3)$ \\
\hline Diameter of resection specimen, mean (range), mm & $24(12-40)$ \\
\hline Histology, $\mathrm{n}(\%)$ & $18 / 24(75.0)$ \\
\hline Complete resection (R0) & $21 / 24(87.5)$ \\
\hline Full-thickness resection & \\
\hline Adverse events, $\mathrm{n}(\%)$ & $1(4.0)$ \\
\hline Minor bleeding & $0(0)$ \\
\hline Requirement for blood transfusion & $0(0)$ \\
\hline Perforation & $0(0)$ \\
\hline Need for emergency surgery & $2(8.0)$ \\
\hline Postpolypectomy syndrome & $4(1-12)$ \\
\hline Duration of hospital stay, mean (range), days & $127(43-372)$ \\
\hline Follow-up & $4(16)$ \\
\hline Duration, mean (range), days & $1(4)$ \\
\hline Residual adenoma, $\mathrm{n}$ & \\
\hline Local recurrence, $\mathrm{n}$ &
\end{tabular}

FTRD, full-thickness resection device.

show any residual adenoma at follow-up after 3 months. Two patients with recurrent adenomas in the rectum and in the ascending colon had small residual adenomas (max. $5 \mathrm{~mm}$ ) at 6 months' follow-up, which were successfully resected using a snare. Further follow-up did not show any residual or recurrent adenoma.

\section{Discussion}

The majority of advanced, colonic, nonpedunculated neoplasms can be treated effectively by modern resection techniques such as EMR or ESD. However, endoscopic resection of recurrent nonlifting (or previously untreated) lesions remains a challenge. ESD has been shown to be feasible for such lesions, but the perforation rate has been reported to be up to $14.7 \%$ [2,3]. Moreover, ESD is technically demanding, requires a substantial amount of training, and is time consuming even in experienced hands. EFTR using a clip-and-cut technique may offer a simpler and time-efficient modality for the treatment of these lesions. Resection with the FTRD can be performed by endoscopists with experience in OTSC application and advanced resection techniques. The median procedure time in this study was 50 minutes including the marking, resection and re-inspection; the resection procedure itself takes about 10 minutes. Compared with EMR or ESD, the diagnostic yield of EFTR may be higher because the pathologist receives a full-thickness (en bloc) resection specimen. In cases of T1 carcinomas, this allows exact determination of submucosal infiltration and R0 status. For this situation, the FTRD can be used as a simple diagnostic tool or for tissue acquisition, and may also be therapeutic in low risk situations. T1 carcinomas with known high risk features such as poor differentiation or deep submucosal infiltration ( $>1000 \mu \mathrm{m})$ should, of course, not be treated with EFTR as these lesions definitively require oncological resection.

Apart from the indications mentioned above, EFTR can also be used for resection of adenomas at difficult anatomic locations, such as adenomas involving a diverticulum [13] or adenomas arising from the appendiceal orifice. In these cases, EFTR may be a minimally invasive and potentially more cost-effective alternative to surgical therapy. Another indication for EFTR is resection of small subepithelial tumors (e.g. neuroendocrine tumors) in the lower GI tract. Moreover, the FTRD can be used for acquisition 
of a full-thickness specimen in patients with suspected colonic motility disorders, such as Hirschsprung's disease. Compared with conventional full-thickness biopsies, a large resection specimen may facilitate histological diagnosis of aganglionosis in these patients.

The effectiveness of OTSCs for closure of GI perforations and leakages has been well investigated [14-16]. The concept of using a conventional OTSC for defect closure after full-thickness resection has been shown to be feasible in porcine colonic experiments and in a recent clinical study investigating EFTR of gastric subepithelial gastric tumors $[17,18]$. In contrast to post-EFTR wall closure with conventional through-the-scope clips or T-tags $[19,20]$, the OTSC allows single-step defect closure, which may be more time efficient than a step-by-step procedure. In contrast to secondary closure of the defect after EFTR, securing GI wall patency before resection may be a safer approach, as contamination of the abdominal cavity by colonic contents can be avoided [21, 22]. Moreover, a secure GI wall closure prior to resection would render laparoscopic assistance (as described in the study by Schlag et al. [18]) unnecessary.

Resection of lesions in the upper GI tract and in the colorectum by application of a conventional 11/6t OTSC followed by snare resection was recently shown to be feasible [5-7]. The main difference with the FTRD is the preloaded snare in the cap, which allows one-step resection immediately after OTSC deployment, leading to further simplification and acceleration of the procedure. The FTRD cap is equipped with a modified 14-mm OTSC and enables the capture of more tissue, as it is longer $(23 \mathrm{~mm})$ and wider ( $13 \mathrm{~mm}$ diameter). The amount of tissue that can be pulled into the cap determines the size of the resection. In preclinical porcine experiments, the maximum size of colonic resection specimens was $30 \times 30$ and $40 \times 42 \mathrm{~mm}[8,11]$; one recent study even reported an average diameter of $54 \mathrm{~mm}$ [9].

However, these experiments were performed on healthy colonic tissue. Thickness, rigidity, and mobility of the colonic wall may differ in "real life" and strongly depend on anatomic location and presence of fibrosis/inflammation caused by pre-treatment. This is indeed reflected by the mean size of the resection specimen in the current study, which was $24 \mathrm{~mm}$ (range $12-40 \mathrm{~mm}$ ). On the basis of the current data, we would not recommend the use of the FTRD for the resection of lesions $>30 \mathrm{~mm}$. In cases of severe scaring or inflammation, the maximum size of the lesion may be even less $(20-25 \mathrm{~mm})$. Rigidity and mobility of the GI wall also influences resection depth. In the current series, fullthickness resection of the colonic wall could not be confirmed histologically in three patients. This was due to fibrosis in two cases and to limited mobility of the lower rectal wall in one case. In general, the ability of the FTRD to achieve real full-thickness resection in the lower rectum may be limited because of fixation of the rectal wall in the perirectal tissue. However, it can be used to achieve a deep rectal resection, which may be sufficient depending on indication. Compared with transanal endoscopic microsurgery, the advantage of the FTRD is that it can be used to target lesions in the entire colon, as shown in the current cohort.

One drawback of the device is that its long cap limits endoscopic view and flexibility of the endoscope tip. In some cases, this may hamper the advance of the endoscope through difficult anatomic lesions such as the sigmoid or colonic flexures. Although a balloon had to be used in the current study to achieve sigmoid passage in two patients, 24 out of 25 target lesions were reached successfully. These included 10 lesions in the right-sided colon, which demonstrates the applicability of the device in all colonic locations.

This study exclusively reports on resections in the lower GI tract. Application of the FTRD for gastric full-thickness resection may not be possible as a result of the thickness of the gastric wall. Moreover, in our experience, the outer diameter of the device $(21 \mathrm{~mm})$ compromises successful transoral insertion. Consequently, the device is currently only approved for application in the lower GI tract.

Another limitation of the device is that the lateral margins of the lesion are not easily visible circumferentially during retraction of the lesion into the cap. To avoid this, it is crucial to grasp the lesion at its center in order to ensure that the whole circumference is incorporated into the cap. Moreover, thorough bowel preparation is crucial in order to achieve good visibility of the lesion. This, together with the fact that the lesion may not have been retracted far enough into the cap, may have accounted for the macroscopically incomplete resections in the current study. In general, the main factor determining resection success is the ability to pull the lesion completely into the cap.This strongly depends on thickness and rigidity of the colonic wall, which is determined by the degree of fibrosis and the presence of inflammation. However, the degree of fibrosis cannot be assessed precisely before resection, and it is usually quite obvious during the procedure if the lesion cannot be completely incorporated into the cap.In such cases, it may be better to stop the FTRD procedure and to consider an alternative (possibly surgical) therapy.

Once the OTSC is deployed, it may hamper further follow-up and re-treatment in cases of incomplete resection. However, combination use with a novel endoscopic cutting device to remove the clip $[23,24]$ may facilitate repeat procedures and further endoscopic treatment. To allow sufficient healing of the colonic wall defect, in our experience the clip should not be removed before 8 weeks after EFTR. In cases of remnant or recurrent adenoma after clip removal, EFTR with the FTRD can again be applied if there is not too much scarring.

Although not observed in the current patient cohort, a potential risk of the EFTR procedure may be accidental clipping of extracolonic structures (e.g. vessels or small bowel). To avoid unintended incorporation of organs next to the colonic wall, it is crucial to exclusively use traction with the forceps or the tissue anchor to pull the target lesion into the cap. Suction should be avoided completely or, if really necessary, should be used only very gently.

In the current study, five patients with adenomas at the appendix were included. All resections involved the complete appendiceal orifice. As the snare resection is performed "above" the OTSC, opening of the appendix into the abdominal cavity may not be a major concern. However, clipping of the orifice may, at least theoretically, lead to complications such as appendicitis or development of a mucocele. Apart from two patients who developed postpolypectomy syndrome, which was successfully treated with antibiotics, no severe complications were observed. However, due to the very limited experience at this early stage of clinical use, resections at this special anatomic location may not be considered as a standard procedure. We would strongly recommend that such patients are treated exclusively within clinical studies and only after thorough discussion of alternative surgical resection with the patient.

In conclusion, the novel over-the-scope device is a valuable and powerful tool for endoscopic full-thickness resection. It can be used for a variety of diagnostic and therapeutic indications in 
the lower GI tract. Future studies are needed to further investigate clinical use and applicability of the device.

Competing interests: The Department of Gastroenterology and Oncology has received financial support from Ovesco Endoscopy for the coordination and performance of a multicenter trial investigating treatment of recurrent peptic ulcer bleeding using overthe-scope clips. Prof. Caca and Dr. Schmidt have received lecture fees from Ovesco Endoscopy for FTRD training courses.

\section{Acknowledgment}

We thank Ovesco Endoscopy for kindly providing the resection device and for the excellent technical support. We also thank our endoscopy nurse team for assistance during the procedures.

\section{References}

1 Maguire LH, Shellito PC. Endoscopic piecemeal resection of large colorectal polyps with long-term followup. Surg Endosc 2014; 28: 2641 2648

2 Kuroki Y, Hoteya S, Mitani T et al. Endoscopic submucosal dissection for residual/locally recurrent lesions after endoscopic therapy for colorectal tumors. J Gastroenterol Hepatol 2010; 25: 1747-1753

3 Sakamoto T, Saito Y, Matsuda T et al. Treatment strategy for recurrent or residual colorectal tumors after endoscopic resection. Surg Endosc 2011; 25: 255-260

4 Moss A, Williams SJ, Hourigan LF et al. Long-term adenoma recurrence following wide-field endoscopic mucosal resection (WF-EMR) for advanced colonic mucosal neoplasia is infrequent: results and risk factors in 1000 cases from the Australian Colonic EMR (ACE) study. Gut 2015; 64: 57-65

5 Mönkemüller K, Peter S, Toshniwal J et al. Multipurpose use of the 'bear claw' (over-the-scope-clip system) to treat endoluminal gastrointestinal disorders. Dig Endosc 2014; 26: 350 - 357

6 Sarker S, Gutierrez JP, Council L et al. Over-the-scope clip-assisted method for resection of full-thickness submucosal lesions of the gastrointestinal tract. Endoscopy 2014; 46: 758 - 761

7 Fähndrich M, Sandmann M. Endoscopic full-thickness resection for gastrointestinal lesions using the over-the-scope clip system: a case series. Endoscopy 2015; 47: 76 - 79

8 Schurr MO, Baur F, Ho CN et al. Endoluminal full-thickness resection of GI lesions: a new device and technique. Minim Invasive Ther Allied Technol 2011; 20: 189-192
9 Schurr MO, Baur FE, Krautwald M et al. Endoscopic full-thickness resection and clip defect closure in the colon with the new FTRD system: experimental study. In press 2015. Surg Endosc DOI 10.1007/s00464014-3923-X

10 von Renteln D, Rösch T, Kratt T et al. Endoscopic full-thickness resection of submucosal gastric tumors. Dig Dis Sci 2012; 57: 1298 - 1303

11 von Renteln D, Kratt T, Rösch T et al. Endoscopic full-thickness resection in the colon by using a clip-and-cut technique: an animal study. Gastrointest Endosc 2011; 74: 1108-1114

12 Schmidt A, Damm M, Caca K. Endoscopic full thickness resection using a novel over-the-scope device. Gastroenterolgy 2014; 47: 740-742

13 Valli PV, Kaufmann M, Vrugt B et al. Endoscopic resection of a diverticulum-arisen colonic adenoma using a full-thickness resection device. Gastroenterology 2014; 147: 969-971

14 Weiland T, Fehlker M, Gottwald T et al. Performance of the OTSC system in the endoscopic closure of iatrogenic gastrointestinal perforations: a systematic review. Surg Endosc 2013; 27: 2258 - 2274

15 Voermans RP1st, Le MoineO, von Renteln D et al. Efficacy of endoscopic closure of acute perforations of the gastrointestinal tract. Clin Gastroenterol Hepatol 2012; 10: 603-608

16 von Renteln D, Schmidt A, Vassiliou MC et al. Endoscopic closure of large colonic perforations using an over-the-scope clip: a randomized controlled porcine study. Endoscopy 2009; 41: 481-486

17 von Renteln D, Schmidt A, Vassiliou MC et al. Endoscopic full-thickness resection and defect closure in the colon. Gastrointest Endosc 2010; 71: $1267-1273$

18 Schlag $C$, Wilhelm D, von Delius $S$ et al. EndoResect study: endoscopic full-thickness resection of gastric subepithelial tumors. Endoscopy 2013; 45: 4-11

$19 \mathrm{Xu}$ M, Wang XY, Zhou PH et al. Endoscopic full-thickness resection of colonic submucosal tumors originating from the muscularis propria: an evolving therapeutic strategy. Endoscopy 2013; 45: 770-773

20 Raju GS, Malhotra A, Ahmed I. Colonoscopic full-thickness resection of the colon in a porcine model as a prelude to endoscopic surgery of difficult colon polyps: a novel technique (with videos). Gastrointest Endosc 2009; 70: 159-165

21 Kopelman Y, Siersema PD, Bapaye A et al. Endoscopic full-thickness Gl wall resection: current status. Gastrointest Endosc 2012; 75: 165-173

22 Kantsevoy SV. Endoscopic full-thickness resection: new minimally invasive therapeutic alternative for GI-tract lesions. Gastrointest Endosc 2006; 64: $90-91$

23 Schmidt A, Riecken B, Damm M et al. Endoscopic removal of over-thescope clips using a novel cutting device: a retrospective case series. Endoscopy 2014; 46: 762 - 766

24 Schostek S, Ho CN, Melbert M et al. DC current pulses for OTSC clip fragmentation: technology and experimental study. In press 2015. Surg Endosc DOI 10.1007/s00464-014-3935-6 